\title{
Excited states of the water molecule: Analysis of the valence and Rydberg character
}

\author{
Mercedes Rubio, ${ }^{1,2, a)}$ Luis Serrano-Andrés, ${ }^{1}$ and Manuela Merchán ${ }^{1}$ \\ ${ }^{1}$ Instituto de Ciencia Molecular, Universitat de València, Apartado 22085, ES-46071 Valencia, Spain \\ ${ }^{2}$ Fundació General de la Universitat de València, Plaza del Patriarca, 4, 1 , ES-46002 Valencia, Spain
}

(Received 8 November 2007; accepted 3 January 2008; published online 12 March 2008)

\begin{abstract}
The excited states of the water molecule have been analyzed by using the extended quantum-chemical multistate CASPT2 method, namely, MS-CASPT2, in conjunction with large one-electron basis sets of atomic natural orbital type. The study includes 13 singlet and triplet excited states, both valence and $3 s-, 3 p$-, and $3 d$-members of the Rydberg series converging to the lowest ionization potential and the $3 s$ - and $3 p$-Rydberg members converging to the second low-lying state of the cation, $1^{2} A_{1}$. The research has been focused on the analysis of the valence or Rydberg character of the low-lying states. The computation of the $1{ }^{1} B_{1}$ state of water at different geometries indicates that it has a predominant $3 s$-Rydberg character at the equilibrium geometry of the molecule but it becomes progressively a valence state described mainly by the one-electron $1 b_{1}$ $\rightarrow 4 a_{1}$ promotion, as expected from a textbook of general chemistry, upon elongation of the $\mathrm{O}-\mathrm{H}$ bonds. The described valence-Rydberg mixing is established to be originated by a molecular orbital (MO) Rydbergization process, as suggested earlier by R. S. Mulliken [Acc. Chem. Res. 9, 7 (1976)]. The same phenomenon occurs also for the $1{ }^{1} A_{2}$ state whereas a more complex behavior has been determined for the $2{ }^{1} A_{1}$ state, where both MO Rydbergization and configurational mixing take place. Similar conclusions have been obtained for the triplet states of the molecule. (C) 2008 American Institute of Physics.
\end{abstract}

[DOI: $10.1063 / 1.2837827$ ]

\section{INTRODUCTION}

Although the spectroscopic properties of the water molecule have been studied since long time ago ${ }^{1,2}$ they are still subject of current interest, ${ }^{3-8}$ greatly motivated by the chemical and biological importance of the system. In particular, the characterization of the low-lying electronic excited states as valence or Rydberg is an open debate which requires a careful theoretical analysis from the viewpoint of modern $a b$ initio quantum chemistry. A number of earlier studies reported the absorption and photoionization cross sections of water vapor in the vacuum ultraviolet region, ${ }^{2,9-13}$ (see also Ref. 14 for a review of the earlier work), as well as excitation energies obtained by electron-impact spectroscopy. ${ }^{15,16}$ The observed bands were interpreted as Rydberg series on the basis of the quantum defect analysis and using available $a b$ initio calculations as a guide for the assignments. ${ }^{11-13,16-19}$ A comprehensive $a b$ initio study on the excited states of water performed in 1974 by Goddard and $\mathrm{Hunt}^{17}$ characterized all the computed states below $11.7 \mathrm{eV}$ as having Rydberg nature, a result supported at the configuration interaction (CI) level. ${ }^{18,19}$ As shall be discussed below, the electronic spectrum of water in the gas phase is currently interpreted as composed either of different Rydberg series or implying excited states with a significant Rydberg character at the ground-state equilibrium geometry (see, for instance, Ref. 20 for a more recent review). How can this scenario

\footnotetext{
a) Author to whom correspondence should be addressed. Electronic mail: mercedes.rubio@uv.es.
}

conciliate with our simple knowledge of the electronic structure of the water molecule? It is obvious that the ground state of the system can be described as having two valence unoccupied antibonding molecular orbitals $\left(4 a_{1}\right.$ and $2 b_{2}$ in $C_{2 v}$ symmetry), and corresponding valence excited states could be expected. The present study attempts to throw more light into this aspect of the excited states of water which we consider has been somewhat overlooked. ${ }^{11,14,21-23}$

Despite earlier conclusions which emphasized the Rydberg character of the band, ${ }^{17-19}$ the experimental evidence does suggest some valence character for the lowest-energy band of the gas-phase electronic spectrum of water. ${ }^{11,14,23}$ The band is broad $(6.8-8.2 \mathrm{eV})$, with poorly defined vibrational progressions, and has its maximum absorption around $7.4 \mathrm{eV}{ }^{4}$ These features result from direct photodissociation of water into ground-state $\mathrm{H}$. and $\mathrm{OH}$. radicals, as shown experimentally ${ }^{24,25}$ and theoretically. ${ }^{26}$ There is a unanimous agreement in assigning the lowest-energy band to the $1{ }^{1} B_{1}$ state, usually named $\tilde{A}^{1} B_{1}$ state according to Herzberg's notation, ${ }^{27}$ but not in the valence, Rydberg, or mixed valence-Rydberg nature of the state. As far as we know, only Wang et al. ${ }^{11}$ claimed a significant valence character, ascribed to the one-electron $1 b_{1} \rightarrow 4 a_{1}$ promotion, for the band at $7.4 \mathrm{eV}$ and proposed a different location for the related ${ }^{1} B_{1} \quad\left(1 b_{1} \rightarrow 3 s\right)$ Rydberg state. In contrast, according to Robin $^{14}$ and using his own words: "the valence $1 b_{1} \rightarrow 4 a_{1}$ configuration is totally dissolved in the conjugated Rydberg sea," and therefore none of the excited states of water could be considered as having valence nature. The lowest-energy 
$(n=3)$ member of the $1 b_{1} \rightarrow n s$ Rydberg series was, however, predicted to have the largest contribution of the $4 a_{1}$ valence orbital $^{22}$ in line with experimental evidence. ${ }^{11,14}$ A somewhat different point of view (see Ref. 14 for discussion) was provided by Mulliken, who described the $1{ }^{1} B_{1}$ state of water as a semi-Rydberg or near-Rydberg state meaning that there is only one state described by a valence-Rydberg mixing in the wave function, a character which is in addition affected by the internuclear distance. ${ }^{21,28}$ Moreover, Mulliken explained the change in the nature of the state with the internuclear distance as a result of the Rydbergization of the molecular orbital (MO) where the electron is excited to; namely, the orbital corresponds to a $3 s$-Rydberg $\mathrm{MO}$ at short $\mathrm{O}-\mathrm{H}$ distances and close to the ground-state equilibrium geometry, increasing its valence character $\left(4 a_{1}\right)$ when elongating the $\mathrm{O}-\mathrm{H}$ bond. To our knowledge, the composition of the $3 s / 4 a_{1}$ orbital as a function of the $\mathrm{O}-\mathrm{H}$ distance was analyzed only in an earlier study performed by Horsley and Fink. ${ }^{29}$ Chergui and Schwentner ${ }^{30}$ also attempted to show experimentally the MO Rydbergization postulated by Mulliken for the $1{ }^{1} B_{1}$ of water. On the other hand, on the basis of the different shape of the bands recorded when studying photodissociation channels of water, Dutuit et al. ${ }^{23}$ proposed a valence character for the $n s a_{1}$-Rydberg series. Furthermore, they attributed the valence character of the $3 s a_{1}$-Rydberg states to MO Rydbergization as described by Mulliken. ${ }^{21,28}$

In the last decade, different theoretical studies on the excited states of water have been carried out using coupledcluster methods, ${ }^{6,31}$ density functional theory (DFT) approaches, 5,32 and multireference CI techniques. ${ }^{33}$ Some of the studies ${ }^{31,34}$ relate the contribution of the antibonding valence $4 a_{1}$ orbital to the lowest singlet excited state, $\widetilde{A}^{1} B_{1}$, to the dissociative nature of the state. ${ }^{24,25}$ However, the character of the low-lying excited states as function of the $\mathrm{O}-\mathrm{H}$ internuclear distance has not been analyzed and theoretical efforts $^{32,33}$ have been mainly focused on determining the low-lying potential energy surfaces of water related to photodissociation processes of the molecule. We report here a high-level $a b$ initio study of the singlet and triplet excited states of water by using multiconfigurational second-order perturbation theory through the CASPT2 method. ${ }^{35,36}$ This methodology is nowadays a well-established approach to accurately compute excited states in different types of compounds (see, for instance, Refs. 37-40). Furthermore, the CASPT2 method is particularly suited for the characterization of potential energy surfaces because of the inherent flexibility of the complete active space self-consistent field (CASSCF) wave function used as reference in the secondorder perturbation treatment. ${ }^{41}$ To the best of our knowledge, only results for the $1{ }^{1} B_{1}$ and $1{ }^{3} B_{1}$ states have been previously reported at the CASPT2 level, but the analysis was mainly focused on their electric properties. ${ }^{42}$ The present study provides accurate excitation energies for the singlet and triplet excited states of the water molecule at the groundstate structure and at several geometries along the symmetrical stretching dissociation channel, paying special attention to the nature of the excited states. In the light of these results, we expect to achieve a better understanding of the Rydberg/ valence character of the electronic spectrum of gas-phase water. Such knowledge is crucial for rationalizing the observed blueshift of the absorption spectrum of liquid water and ice since Rydberg and valence states are differently affected in condensed phases. ${ }^{14}$

\section{METHODS AND CALCULATION DETAILS}

In order to study the Franck-Condon $(\mathrm{FC})$ region of the molecule, calculations were performed at the experimental equilibrium geometry of the ground state reported by Herzberg $\quad\left(r_{\mathrm{O}-\mathrm{H}}=0.958 \AA\right.$ and $<\mathrm{HOH}=104.5^{\circ}, \quad C_{2 v}$ symmetry). ${ }^{27}$ According to the standard orientation, the molecule was placed in the $y z$ plane with the $z$ axis corresponding to the $C_{2}$ symmetry axis. In order to analyze the character of the electronic states at different molecular geometries, potential energy curves obtained by symmetrically stretching the $\mathrm{O}-\mathrm{H}$ bond lengths have also been computed. Generally contracted basis sets of the atomic natural orbital (ANO-L) type were used, which were built from the $\mathrm{O}(14 s 9 p 4 d 3 f) / \mathrm{H}(8 s 4 p 3 d)$ primitive sets. ${ }^{43}$ These basis sets were designed to optimally treat correlation and polarization effects. In order to select the contraction scheme to be used, the vertical excitation energy to the $1{ }^{1} B_{1}$ state was computed at the CASPT2 level using the full valence active space and several basis sets. The results are collected in Table I together with the values of the second moments of the electronic charge distribution computed at the CASSCF level. To properly describe Rydberg states, the ANO-L type basis sets were supplemented with two $s$ (exponents 0.02348 and 0.00822 ), two $p$ (exponents 0.01579 and 0.005 53), and one or two $d$ (exponents 0.05627 and 0.01970 ) diffuse functions placed at the oxygen atom position. The exponents of the diffuse functions were determined by an even-tempered expansion from the primitive sets. As shown in Table I, the ANO-L $\mathrm{O}[5 s 4 p 1 d] / \mathrm{H}[2 s 1 p]$ contraction (basically a quadruple- and double-zeta plus polarization scheme for oxygen and hydrogen, respectively) yields a transition energy for the $1{ }^{1} B_{1}$ state differing $\pm 0.02 \mathrm{eV}$ from that obtained with larger basis sets, including up to $f$ functions, and more flexible contraction schemes. The addition of diffuse functions has also a small effect on the computed excitation energy and second moments. It is worth noting that the $1{ }^{1} B_{1}$ state is qualitatively well described even with the smallest generally contracted ANO-L basis sets but not with the standard 6-31G $(d, p)$ basis set, with the $1{ }^{1} B_{1}$ state placed vertically at 7.79 and $8.68 \mathrm{eV}$, respectively. The latter is more than $1 \mathrm{eV}$ higher than the experimental datum, $7.45 \mathrm{eV} .{ }^{4}$ Taking into account the calibration calculations collected in Table I, the $\mathrm{O}[5 s 4 p 2 d 1 f] / \mathrm{H}[3 s 2 p 1 d]+(2 s 2 p 2 d)$ basis set was used for the calculation of vertical excitation energies, while the smaller $\mathrm{O}[5 s 4 p 1 d] / \mathrm{H}[2 s 1 p]+(2 s 2 p 1 d)$ basis set was employed in the determination of the stretching potential energy curves of the low-lying excited states (basis set: VII and $\mathrm{V}$, respectively, according to the notation used in Table I).

Multiconfigurational wave functions were initially determined at the CASSCF level. ${ }^{41}$ In all calculations, the oxygen $1 s$ electrons were inactive and all the valence orbitals were included in the active space. The full valence active space 
TABLE I. CASPT2 vertical excitation energies (eV) and CASSCF second moments (a.u.) computed for the $1{ }^{1} B_{1}$ state with different basis sets. Calculations at the experimental ground-state geometry. The full valence active space is used in the calculations.

\begin{tabular}{lccccc}
\hline \hline Basis set $^{\text {a }}$ & CASPT2 & $\left\langle x^{2}\right\rangle$ & $\left\langle y^{2}\right\rangle$ & $\left\langle z^{2}\right\rangle$ & $\left\langle r^{2}\right\rangle$ \\
\hline I: $6-31 \mathrm{G}(d, p)$ & 8.68 & 5.4 & 6.6 & 6.8 & 18.8 \\
II: $[3 s 2 p 1 d] /[2 s 1 p]$ & 7.79 & 9.1 & 9.7 & 10.3 & 29.1 \\
III: $[4 s 3 p 1 d] /[2 s 1 p]$ & 7.66 & 9.2 & 10.1 & 10.8 & 30.1 \\
IV: $[5 s 4 p 1 d] /[2 s 1 p]$ & 7.49 & 9.2 & 9.7 & 10.7 & 29.6 \\
V: $[5 s 4 p 1 d] /[2 s 1 p]+s, s^{\prime}+p, p^{\prime}+d$ & 7.43 & 9.7 & 10.4 & 12.6 & 32.7 \\
VI: $[5 s 4 p 2 d 1 f] /[3 s 2 p 1 d]$ & 7.48 & 9.8 & 10.3 & 11.6 & 31.7 \\
VII: $[5 s 4 p 2 d 1 f] /[3 s 2 p 1 d]+s, s^{\prime}+p, p^{\prime}+d, d^{\prime}$ & 7.48 & 9.4 & 10.2 & 12.3 & 31.9 \\
VII: $[6 s 5 p 3 d 2 f] /[3 s 2 p 1 d]$ & 7.51 & 9.7 & 10.2 & 11.7 & 31.6 \\
Expt. ${ }^{\text {b } 7.447 \mathrm{eV}}$ & & & & &
\end{tabular}

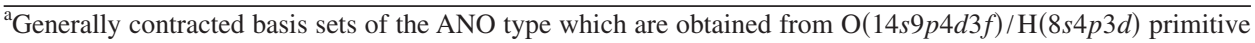
sets. When indicated, the basis set is supplemented with two $s$ (exponents 0.02348 and 0.00822 ), two $p$ (exponents 0.01579 and 0.00553 ), and one or two $d$ (exponents 0.05627 and 0.01970 ) diffuse functions placed at the oxygen atom position, which are constructed from an even-tempered expansion from the primitive set.

${ }^{\mathrm{b}}$ High resolution absorption spectrum from Ref. 4. Band maximum.

comprises six orbitals with eight electrons and corresponds to (3120) where the entries stand for the number of active orbitals belonging to the irreducible representations $a_{1}, b_{1}$, $b_{2}$, and $a_{2}$, respectively, of the $C_{2 v}$ point group. Rydberg orbitals have to be added to the valence active space in order to have enough flexibility for computing all types (Rydberg, valence, or mixed valence-Rydberg) of excited states. With the simultaneous inclusion of the $3 s-, 3 p-$, and $3 d$-Rydberg orbitals (nine orbitals), the active space becomes unnecessarily large. Therefore, selected Rydberg orbitals were added to the full valence active space according to the symmetry of the state and taking into account that we are interested in excitations from the two highest occupied molecular orbitals $\left(1 b_{1}\right.$ and $\left.3 a_{1}\right)$. For all the states, the computed vertical excitation energies were checked with respect to further enlargements of the active space and stable results $( \pm 0.1 \mathrm{eV})$ were found. The molecular orbitals for the excited states were obtained from state-average (SA) CASSCF computations. Table II compiles the final active spaces employed for the calculation of the singlet and triplet excited states of water and the number of states included in the SA CASSCF calcu-

TABLE II. Details of the CASSCF wave functions used for the calculation of the considered excited states of water.

\begin{tabular}{lll}
\hline \hline State & Active space $^{\mathrm{a}}$ & $N_{\text {states }} \mathrm{b}^{\mathrm{b}}$ \\
\hline${ }^{1,3} A_{1}\left(3 a_{1} \rightarrow 4 a_{1}, 3 s ; 1 b_{1} \rightarrow 3 p_{x}\right)$ & $(4220)$ & 3 \\
${ }^{1,3} A_{1}\left(1 b_{1} \rightarrow 3 d_{x z}\right)$ & $(3320)$ & 4 (singlet), 3 (triplet) \\
${ }^{1,3} A_{1}\left(3 a_{1} \rightarrow 3 p_{z}\right)$ & $(5120)$ & 4 \\
${ }^{1,3} B_{1}\left(1 b_{1} \rightarrow 4 a_{1}, 3 s, 3 p_{z}\right)$ & $(5120)$ & 3 \\
${ }^{1,3} B_{1}\left(1 b_{1} \rightarrow 3 d_{z}^{2}, 3 d_{x}^{2}, y^{2}\right)$ & $(7120)$ & 5 \\
${ }^{1,3} B_{1}\left(3 a_{1} \rightarrow 3 p_{x}\right)$ & $(3220)$ & 2 \\
${ }^{1,3} B_{2}\left(1 b_{1} \rightarrow 3 d_{x y} ; 3 a_{1} \rightarrow 2 b_{2}, 3 p_{y}\right)$ & $(3141)$ & 2 \\
${ }^{1,3} A_{2}\left(1 b_{1} \rightarrow 2 b_{2}, 3 p_{y}, 3 d_{y z}\right)$ & $(3141)$ & 3 \\
\hline \hline
\end{tabular}

$\overline{\bar{a} \text { Number of active orbitals of the irreducible representations of the } C_{2 v} \text { point }}$ group $\left(a_{1}, b_{1}, b_{2}, a_{2}\right)$.

${ }^{\mathrm{b}}$ Number of roots included in the corresponding state-average CASSCF calculation. lations. The transition energies are referred to the ground state computed with the same active space as the excited state.

To take into account the remaining (dynamic) correlation effects, the CASSCF wave functions were employed as reference functions in a single-state second-order perturbation CASPT2 treatment. ${ }^{35,36}$ The oxygen $1 s$ electrons were kept frozen at this calculation level. In addition, the so-called imaginary level-shift technique ${ }^{44}$ was used with a shift value of 0.2 a.u. in order to avoid the possible presence of weakly interacting intruder states. The coupling of the CASSCF wave functions via dynamic correlation was dealt by using the extended multistate CASPT2 approach, the MS-CASPT2 method. ${ }^{45}$ An effective Hamiltonian matrix is constructed where the diagonal elements correspond to the CASPT2 energies and the off-diagonal elements introduce the coupling to second order in the dynamic correlation energy. In this way, all the states of a given symmetry are allowed to interact under the influence of dynamic correlation, and the possible erratic valence-Rydberg mixing can be removed (see, for instance, Ref. 46). The linear combination of the CASSCF states produced by the MS-CASPT2 calculation is named perturbatively modified CAS (PMCAS) CI wave function. ${ }^{45}$ Properties of the states were determined from the PMCAS-CI wave functions. The oscillator strengths were computed using PMCAS-CI transition dipole moments according to the CASSCF state interaction (CASSI) $\operatorname{method}^{47,48}$ and energy differences obtained at the MSCASPT2 level. All calculations were performed with the MOLCAS-6 quantum-chemistry software. ${ }^{49}$

\section{RESULTS AND DISCUSSION}

In $C_{2 v}$ symmetry the ground state of the water molecule is mainly described by the electronic configuration $\left(1 a_{1}\right)^{2}\left(2 a_{1}\right)^{2}\left(1 b_{2}\right)^{2}\left(3 a_{1}\right)^{2}\left(1 b_{1}\right)^{2}$ where the $1 a_{1}$ orbital corresponds to the $1 s$ orbital of the oxygen atom. The valence orbitals comprise the four highest occupied orbitals listed above and two unoccupied antibonding orbitals of $a_{1}$ and $b_{2}$ 
TABLE III. Computed MS-CASPT2 vertical transition energies $(\Delta E, \mathrm{eV})$, oscillator strengths $(f)$, and second moments (from PMCAS-CI wave functions) (a.u.) for the singlet excited states of the water molecule at the ground-state equilibrium geometry.

\begin{tabular}{lccrrrrl}
\hline \hline State & \multicolumn{1}{c}{$\Delta E$} & $f$ & $\left\langle x^{2}\right\rangle$ & $\left\langle y^{2}\right\rangle$ & $\left\langle z^{2}\right\rangle$ & $\left\langle r^{2}\right\rangle$ & Expt. $(\mathrm{eV})$ \\
\hline $1{ }^{1} A_{1}$ & & & 5.6 & 3.3 & 4.5 & 13.4 & \\
$1{ }^{1} B_{1}\left(1 b_{1} \rightarrow 3 s / 4 a_{1}\right)$ & 7.50 & 0.033 & 8.9 & 9.8 & 11.4 & 30.1 & $7.4,{ }^{\mathrm{a}} 7.447,{ }^{\mathrm{b}} 7.42^{\mathrm{c}}$ \\
$1{ }^{1} A_{2}\left(1 b_{1} \rightarrow 3 p_{y} / 2 b_{2}\right)$ & 9.27 & Forbidden & 9.8 & 22.2 & 11.2 & 43.2 & $9.1^{\mathrm{a}}$ \\
$2{ }^{1} A_{1}\left(3 a_{1} \rightarrow 3 s / 4 a_{1}\right)$ & 9.86 & 0.032 & 20.1 & 12.2 & 12.7 & 45.0 & $9.7,{ }^{\mathrm{a}} 9.991^{\mathrm{b}}$ \\
$2{ }^{1} B_{1}\left(1 b_{1} \rightarrow 3 p_{z}\right)$ & 9.95 & 0.006 & 16.4 & 16.2 & 31.3 & 63.9 & $10.01,{ }^{\mathrm{a}, \mathrm{b}} 9.99^{\mathrm{c}}$ \\
$3{ }^{1} A_{1}\left(1 b_{1} \rightarrow 3 p_{x}\right)$ & 10.15 & 0.032 & 33.2 & 13.7 & 15.1 & 62.0 & $10.16,{ }^{\mathrm{a}, \mathrm{b}} 10.17^{\mathrm{c}}$ \\
$2{ }^{1} A_{2}\left(1 b_{1} \rightarrow 3 d_{y z}\right)$ & 10.84 & Forbidden & 21.5 & 55.5 & 29.0 & 106.0 & $10.84^{\mathrm{a}}$ \\
${ }^{1} B_{1}\left(1 b_{1} \rightarrow 3 d\right)$ & 11.03 & 0.010 & 22.4 & 48.7 & 28.6 & 99.7 & $10.99^{\mathrm{d}}$ \\
$1{ }^{1} B_{2}\left(1 b_{1} \rightarrow 3 d_{x y}\right)$ & 11.05 & 0.012 & 37.6 & 35.8 & 14.4 & 87.8 & $11.05^{\mathrm{d}}$ \\
${ }^{1} A_{1}\left(1 b_{1} \rightarrow 3 d_{x z}\right)$ & 11.09 & 0.024 & 60.5 & 20.7 & 41.4 & 122.6 & $11.13,{ }^{\mathrm{d}} 11.12^{\mathrm{c}}$ \\
${ }^{1} B_{1}\left(1 b_{1} \rightarrow 3 d\right)$ & 11.19 & 0.015 & 33.4 & 21.0 & 60.8 & 115.2 & \\
$2{ }^{1} B_{2}\left(3 a_{1} \rightarrow 3 p_{y} / 2 b_{2}\right)$ & 11.69 & 0.020 & 13.1 & 29.3 & 12.4 & 54.8 & $11.5^{\mathrm{e}}$ \\
${ }^{1} B_{1}\left(3 a_{1} \rightarrow 3 p_{x}\right)$ & 12.13 & 0.002 & 43.2 & 14.6 & 15.6 & 73.4 & \\
${ }^{1} A_{1}\left(3 a_{1} \rightarrow 3 p_{z}\right)$ & 12.21 & $<0.001$ & 16.5 & 15.3 & 35.7 & 67.5 & \\
\hline \hline
\end{tabular}

${ }^{\mathrm{a} E l e c t r o n-i m p a c t ~ d a t a ~ f r o m ~ C h u t j i a n ~}$ et al. Ref. 16.

${ }^{\mathrm{b}} \mathrm{High}$ resolution vacuum ultraviolet spectrum from Mota et al., Ref. 4.

${ }^{c}$ Wang et al., Ref. 11.

dishiguro et al., Ref. 13.

${ }^{\mathrm{e}}$ Dutuit et al., Ref. 23.

symmetries ( $4 a_{1}$ and $2 b_{2}$ orbitals), as can be derived from a simple qualitative analysis based on symmetry considerations. ${ }^{27}$ The highest occupied molecular orbital, $1 b_{1}$, is a nonbonding orbital closely related to the $2 p_{x}$ orbital of the oxygen atom. This assignment is consistent with the vibrational structure of the lowest-energy band of the photoelectron spectrum. ${ }^{50}$ The $0-0$ feature is the most intense transition of the band, indicating a similar geometry for the neutral molecule and the cation, as could be expected from ionization involving a nonbonding electron. Accordingly, the $1^{2} B_{1}$ state has been computed at the CASPT2 level (valence active space) to lie vertically at $12.54 \mathrm{eV}$ above the ground state, in agreement with both the $0-0$ band position recorded at $12.61 \mathrm{eV}$ (Ref. 50) and the value determined from the convergence of the Rydberg series. ${ }^{9,10}$ The second-energy state of the cation, $1^{2} A_{1}$, arising from removing one electron from the $3 a_{1}$ orbital, has been computed vertically at $14.74 \mathrm{eV}$. Experimental data show a large energy difference $(\sim 0.8 \mathrm{eV})$ between the vertical and adiabatic ionization energies, suggesting therefore an important geometrical molecular reorganization upon ionization, an effect which is consistent with a linear or quasilinear geometry for the $1^{2} A_{1}$ state. ${ }^{50}$ As determined from the band maximum, the second vertical ionization energy is $14.7 \mathrm{eV},{ }^{50}$ matching the present computed value. According to the experimental ionization potentials, ${ }^{50}$ the $1 b_{2}$ orbital is energetically well separated $(>3 \mathrm{eV})$ from the two highest occupied MOs. Therefore, we shall focus the study on the excited states of water arising from excitations from the $3 a_{1}$ and $1 b_{1}$ MOs. The $3 s-, 3 p-$, and $3 d$-members of the singlet and triplet Rydberg series converging to the lowest ionization potential and the $3 s$ - and $3 p$-Rydberg members converging to the $1^{2} A_{1}$ state have been considered. Different types of equilibrium geometries (bent or linear) are expected for the Rydberg states originating from the $1 b_{1}$ and $3 a_{1}$ orbitals, respectively, which can have important consequences in the deactivation pathways of the states. ${ }^{51,52}$ Apart from Rydberg states, from a simple qualitative analysis based on the MOs of the ground state, one could also expect four valence (singlet and triplet) excited states, each one belonging to one of the irreducible representations of the $C_{2 v}$ symmetry group, arising from oneelectron excitations from the two highest occupied MOs to the valence unoccupied $4 a_{1}$ and $2 b_{2}$ orbitals. These possible valence excited states can interact with the corresponding Rydberg states of the same symmetry and close in energy. For instance, the valence singly excited $1 b_{1} \rightarrow 4 a_{1}$ configuration could be mixed with the $1 b_{1} \rightarrow 3 s, 1 b_{1} \rightarrow 3 p_{z}, 1 b_{1}$ $\rightarrow 3 d_{x 2-y 2}$, and $3 d_{z 2}$ Rydberg states, especially with those energetically closer. As outlined in the Introduction, it seems that the valence-Rydberg mixing occurs at the molecular orbital level in the water molecule and results in an electronic spectrum with predominant Rydberg character at the equilibrium ground-state geometry, as confirmed by the present findings (see Table III).

The computed results and selected experimental and previous theoretical information on the singlet excited states of water at the FC geometry are collected in Tables III and IV. The nature of the states is determined from the corresponding PMCAS-CI wave functions and it is shortly described within parentheses in the tables. Thus, the states have been characterized on the basis of the dominant orbital configuration and the expectation values $\left\langle x^{2}\right\rangle,\left\langle y^{2}\right\rangle$, and $\left\langle z^{2}\right\rangle$, which provide an estimate of the diffuseness of the state and the directional distribution of the electronic charge. It is noted that all the states are appreciably more diffuse than the ground state for which the value $\left\langle r^{2}\right\rangle$ is computed to be 13.4 a.u. A detailed analysis of the nature of the states is carried out below. It is also worth mentioning that, except for the $2{ }^{1} A_{1}$ and $3{ }^{1} A_{1}$ states, the computed PMCAS-CI wave functions are similar to those obtained at the CASSCF level. 
TABLE IV. Computed MS-CASPT2 (MS-PT2) vertical excitation energies (eV) for the singlet excited states of the water molecule compared to previous theoretical results.

\begin{tabular}{lrrrrrrr}
\hline \hline State & MS-PT2 & \multicolumn{1}{c}{$\begin{array}{c}\text { MRD- } \\
C^{\mathrm{a}}\end{array}$} & $\begin{array}{r}\text { TD- } \\
\text { DFT }\end{array}$ & $\begin{array}{r}\text { EOM- } \\
\text { CCSD }^{\mathrm{b}}\end{array}$ & ${\text { CCSDR }(3)^{\mathrm{c}}}^{\mathrm{c}}$ & MRCC $^{\mathrm{d}}$ & MRCI+Q $^{\mathrm{e}}$ \\
\hline $1{ }^{1} B_{1}\left(1 b_{1} \rightarrow 3 s / 4 a_{1}\right)$ & 7.50 & 7.63 & 7.61 & 7.58 & 7.66 & 7.51 & 7.81 \\
$1{ }^{1} A_{2}\left(1 b_{1} \rightarrow 3 p_{y} / 2 b_{2}\right)$ & 9.27 & 9.60 & 9.36 & 9.33 & 9.42 & 9.30 & 9.30 \\
$2{ }^{1} A_{1}\left(3 a_{1} \rightarrow 3 s / 4 a_{1}\right)$ & 9.86 & 9.95 & 9.76 & 9.86 & 9.93 & 9.84 & 9.91 \\
$2{ }^{1} B_{1}\left(1 b_{1} \rightarrow 3 p_{z}\right)$ & 9.95 & 10.11 & 10.00 & 9.99 & 10.09 & 10.06 & 10.72 \\
$3{ }^{1} A_{1}\left(1 b_{1} \rightarrow 3 p_{x}\right)$ & 10.15 & 10.11 & 10.23 & 10.21 & 10.28 & 10.19 & 11.26 \\
$2{ }^{1} A_{2}\left(1 b_{1} \rightarrow 3 d_{y z}\right)$ & 10.84 & 11.02 & & & 10.98 & 11.06 & \\
${ }^{1} B_{1}\left(1 b_{1} \rightarrow 3 d\right)$ & 11.03 & & & & & & \\
$1^{1} B_{2}\left(1 b_{1} \rightarrow 3 d_{x y}\right)$ & 11.05 & 11.11 & & & 11.25 & & \\
${ }^{1} A_{1}\left(1 b_{1} \rightarrow 3 d_{x z}\right)$ & 11.09 & 11.16 & & & 11.49 & & \\
${ }^{1} B_{1}\left(1 b_{1} \rightarrow 3 d\right)$ & 11.19 & & & & & & \\
$2{ }^{1} B_{2}\left(3 a_{1} \rightarrow 3 p_{y} / 2 b_{2}\right)$ & 11.69 & & 11.54 & 11.69 & 11.76 & & \\
${ }^{1} B_{1}\left(3 a_{1} \rightarrow 3 p_{x}\right)$ & 12.13 & & & & & & \\
${ }^{1} A_{1}\left(3 a_{1} \rightarrow 3 p_{z}\right)$ & 12.21 & & & & & & \\
\hline \hline
\end{tabular}

${ }^{\mathrm{a}}$ Harrevelt and van Hermert, Ref. 33 .

bd-aug-cc-pVTZ basis set. Cai et al., Ref. 32.

cd-aug-cc-pVQZ basis set, Christiansen et al., Ref. 31.

${ }^{\mathrm{d}} \mathrm{Li}$ and Paldus, Ref. 6.

eaug-cc-pVTZ basis set. Cai et al., Ref. 32 .

Accordingly, the vertical excitation energies computed at the MS-CASPT2 level practically coincide (within $\pm 0.03 \mathrm{eV}$ ) with those obtained at the CASPT2 level. For the $2{ }^{1} A_{1}$ and $3{ }^{1} A_{1}$ states, the coupling via dynamic correlation has been found to be important since both states are found within an energy difference of $0.13 \mathrm{eV}$ at the CASPT2 level. The MS-CASPT2 calculation changes the relative order of these states with respect to that obtained at the CASPT2 level. Moreover, the PMCAS-CI wave functions of the $2{ }^{1} A_{1}$ and $3{ }^{1} A_{1}$ states show a higher degree of configurational mixing involving the singly excited $3 a_{1} \rightarrow 3 s / 4 a_{1}$ and $1 b_{1} \rightarrow 3 p_{x}$ configurations than that obtained for the CASSCF wave functions, in agreement with recent CAS/multireference configuration interaction (MRCI) calculations reported by Borges. ${ }^{8}$ At first glance, the results collected in Table III deviate from the experimental values less than $0.1 \mathrm{eV}$, excluding the computed vertical excitation energies for the three lowest excited states and the $2{ }^{1} B_{2}$ state which show a somewhat larger difference $(0.2 \mathrm{eV})$. In the following, we shall first discuss in detail the results obtained for the singlet excited states in comparison with the experimental data and previous $a b$ initio results. Next, the triplet excited states will be considered.

Singlet excited states. As shown in Table III, the $1{ }^{1} B_{1}$ state is computed to be the lowest singlet excited state of the water molecule, in agreement with previous theoretical results (see Table IV). ${ }^{6,31,32}$ The $1{ }^{1} B_{1}$ state is vertically placed at $7.50 \mathrm{eV}$ and it is related to one of the most intense transitions of the electronic spectrum. The computed oscillator strength of 0.033 has the same order of magnitude as the value (0.049) obtained by Borges ${ }^{7}$ at the CAS/MRCI level and the experimental determinations. ${ }^{53}$ It is well established that the $1{ }^{1} B_{1}$ state, usually called $\tilde{A}$ state, is responsible for the lowest-energy band of the electronic spectrum with maximum at around $7.4 \mathrm{eV} .^{4,11,12,14,16}$ The nature of the $1{ }^{1} B_{1}$ state needs, however, further consideration. The band is broad, covering the energy range $6.8-8.2 \mathrm{eV}$, and has a poorly defined progression which has been related to the symmetric bending mode ${ }^{4,11}$ or to the symmetric stretching vibration on the basis of the results reported by Makochenkawa $e t$ al. ${ }^{54}$ Comparison with the lowest-energy band of the photoelectron spectrum, ${ }^{50}$ corresponding to the removal of a $1 b_{1}$ electron, showed that both bands have different shapes, suggesting therefore some valence character for the $1{ }^{1} B_{1}$ state, according to Robin. ${ }^{14}$ On the contrary, a more detailed analysis of the band at $7.4 \mathrm{eV}$ performed by Wang et al. ${ }^{11}$ led these authors to propose a dominant valence nature for the $1{ }^{1} B_{1}$ state. Furthermore, the corresponding ${ }^{1} B_{1}\left(1 b_{1} \rightarrow 3 s\right)$ state with predominant Rydberg character was tentatively located at $9.08 \mathrm{eV} .{ }^{11}$ The present results, as well as previous theoretical studies (see Table IV), clearly rule out the existence of a ${ }^{1} B_{1}$ state near $9 \mathrm{eV}$ and suggest a valence-Rydberg mixing for the $1{ }^{1} B_{1}$ state. On one hand, considering the computed $\left\langle r^{2}\right\rangle$ values included in Table III, we find that the $1^{1} B_{1}$ state has a diffuse nature $\left(\left\langle r^{2}\right\rangle=30.1\right.$ a.u. $)$, more than twice the value calculated for the ground state but appreciably lower than that obtained for a pure Rydberg state as the $2{ }^{1} B_{1}\left(1 b_{1} \rightarrow 3 p_{z}\right)$ state $\left(\left\langle r^{2}\right\rangle=63.9\right.$ a.u. $)$. As shown in Table I, it is worth recalling that the computed $\left\langle r^{2}\right\rangle$ is quite stable with respect to the inclusion of Rydberg functions in the basis set. On the other hand, the PMCAS-CI wave function computed for the $1{ }^{1} B_{1}$ state is mainly described by the singly excited $1 b_{1} \rightarrow 3 s / 4 a_{1}$ configuration with a weight of $75 \%$. Therefore, the valence-Rydberg character of the state arises mainly from the nature of the excited orbital, labeled here as $3 s / 4 a_{1}$ to illustrate its intermediate character, and not from configurational mixing as seems to suggest the results reported by Diercksen et al. ${ }^{22}$ Thus, we see that the excited orbital is not a pure $3 s$-Rydberg orbital but it has a valence contribution, as reflected by the calculated value for $\left\langle r^{2}\right\rangle$ and it was also obtained in previous theoretical studies. ${ }^{34,55}$ At the ground-state equilibrium geometry, the present MS- 
TABLE V. Computed MS-CASPT2 excitation energies (eV) and other properties for the singlet excited states of symmetrically stretched $\left(r_{\mathrm{O}-\mathrm{H}}=1.4658 \AA\right)$ water. Results from calculations with the basis set $\mathrm{V}$ and active space (5120) for the ${ }^{1} B_{1}$ states, (4220) for the ${ }^{1} A_{1}$ states, and (3141) for the ${ }^{1} A_{2}$ states.

\begin{tabular}{lrrrrr}
\hline \hline State & \multicolumn{1}{c}{$\Delta E^{\mathrm{a}}$} & $\left\langle x^{2}\right\rangle$ & $\left\langle y^{2}\right\rangle$ & $\left\langle z^{2}\right\rangle$ & $\left\langle r^{2}\right\rangle$ \\
\hline $1{ }^{1} B_{1}\left(1 b_{1} \rightarrow 4 a_{1}\right)$ & 7.90 & 5.8 & 5.8 & 6.6 & 18.2 \\
$1{ }^{1} A_{2}\left(1 b_{1} \rightarrow 2 b_{2}\right)$ & 9.11 & 5.1 & 5.9 & 5.8 & 16.8 \\
$2{ }^{1} A_{1}\left(3 a_{1} \rightarrow 4 a_{1}\right)$ & 11.21 & 7.5 & 5.3 & 5.2 & 18.0 \\
$2{ }^{1} B_{1}\left(1 b_{1} \rightarrow 3 s\right)$ & 13.00 & 27.3 & 18.8 & 31.4 & 77.5 \\
$3{ }^{1} B_{1}\left(1 b_{1} \rightarrow 3 p_{z}\right)$ & 13.19 & 17.6 & 14.5 & 32.2 & 64.3 \\
$3{ }^{1} A_{1}\left(1 b_{1} \rightarrow 3 p_{x}\right)$ & 13.22 & 33.1 & 9.8 & 12.6 & 55.5 \\
$2{ }^{1} A_{2}\left(1 b_{1} \rightarrow 3 p_{y}\right)$ & 13.43 & 21.5 & 51.0 & 19.7 & 92.2 \\
\hline \hline
\end{tabular}

${ }^{\mathrm{a}}$ Excitation energies are referred to the ground state computed at the experimental equilibrium geometry $\left(r_{\mathrm{O}-\mathrm{H}}=0.9578 \AA\right)$.

CASPT2 calculations predict only one $1{ }^{1} B_{1}\left(1 b_{1} \rightarrow 3 s / 4 a_{1}\right)$ state of intermediate type. These results strongly support the consideration of the $1^{1} B_{1}$ state of water as a case of MO Rydbergization postulated by Mulliken. ${ }^{21,28}$

In order to get further insight into the nature of the $1{ }^{1} B_{1}$ state, we have analyzed its evolution with respect to the $\mathrm{O}-\mathrm{H}$ internuclear distance. For the sake of simplicity, we have considered the symmetrical stretching of both $\mathrm{O}-\mathrm{H}$ bonds, preserving thus the $C_{2 v}$ symmetry of the system. MSCASPT2 calculations were performed for the three lowest roots of ${ }^{1} B_{1}$ symmetry using the $\mathrm{O}[5 s 4 p 1 d] / \mathrm{H}[2 s 1 p]$ $+(2 s 2 p 1 d)$ basis set (basis set V) and the (5120) active space (full valence active space plus two extra Rydberg orbitals of $a_{1}$ symmetry) at the $C_{2 v}$ geometries with $\mathrm{O}-\mathrm{H}$ distances varying between 0.916 and $1.466 \AA$ and keeping fixed the bond angle to the ground-state equilibrium value $\left(104.5^{\circ}\right)$. The excitation energies and second moments computed for the singlet excited states of water at the $\mathrm{O}-\mathrm{H}$ internuclear distance of $1.466 \AA$ are included in Table V. The computed potential energy curves are shown in Fig. 1 together with the values for $\left\langle r^{2}\right\rangle$, which allow us to characterize the states. The computed vertical excitation energies at the ground-state equilibrium geometry $\left(r_{\mathrm{O}-\mathrm{H}}=0.958 \AA\right)$ are only $0.04-0.1 \mathrm{eV}$ lower than those obtained with the larger basis set VII (see Table III), supporting then the basis set used for building the potential energy curves. As expected from the results collected in Table III, the three low-lying ${ }^{1} B_{1}$ states are related to one of the $1 b_{1} \rightarrow 3 s / 4 a_{1}, 1 b_{1} \rightarrow 3 p_{z}$, and $1 b_{1} \rightarrow 4 s$ oneelectron promotions at geometries with $\mathrm{O}-\mathrm{H}$ bond lengths around the ground-state equilibrium value $(r \sim 0.9-1.0 \AA)$ (see also $\left\langle r^{2}\right\rangle$ values). It should be clarified that the ${ }^{1} B_{1}\left(1 b_{1} \rightarrow 4 s\right)$ state is not included in Table III although it was computed to appear in the energy range studied (vertically located at $10.55 \mathrm{eV}$ with the basis set VII). As stated in the Introduction, the present study is focused on the lowest members $(n=3)$ of the Rydberg series and more diffuse functions should be probably included in the basis set to get accurate results for the $n=4$ members. Our calculations are expected, however, to yield a reliable description for the ${ }^{1} B_{1}\left(1 b_{1} \rightarrow 4 s\right)$ state, which is here considered in order to get a more complete picture of the evolution of the ${ }^{1} B_{1}$ states as function of the $\mathrm{O}-\mathrm{H}$ internuclear distance. As shown in Fig. 1 , the second and third ${ }^{1} B_{1}$ states have Rydberg character at all distances examined since they show high values for $\left\langle r^{2}\right\rangle$ in comparison with the ground state. The type of Rydberg state changes, however, from $1 b_{1} \rightarrow 3 p_{z}$ and $1 b_{1} \rightarrow 4 s$ to $1 b_{1} \rightarrow 3 s$ and $1 b_{1} \rightarrow 3 p_{z}$, respectively, when increasing the $\mathrm{O}-\mathrm{H}$ distances. The shape of the corresponding potential energy curves is similar to the ground-state curve, as could be expected for Rydberg states converging to the ${ }^{2} B_{1}$ cationic state which has a geometry close to the ground-state one. ${ }^{50}$ As can be seen in Fig. 1, the situation is clearly different for the $1{ }^{1} B_{1}$ state. The evolution of the computed $\left\langle r^{2}\right\rangle$ for the $1{ }^{1} B_{1}$ state with the $\mathrm{O}-\mathrm{H}$ distance shows that the state is getting more valence character when stretching the $\mathrm{O}-\mathrm{H}$ bond as a result mainly of the changes undergone by the virtual orbital, in agreement with the concept of Rydbergization. ${ }^{21,28}$ From a simple qualitative description, the energy of the antibonding $4 a_{1}$ orbital decreases when
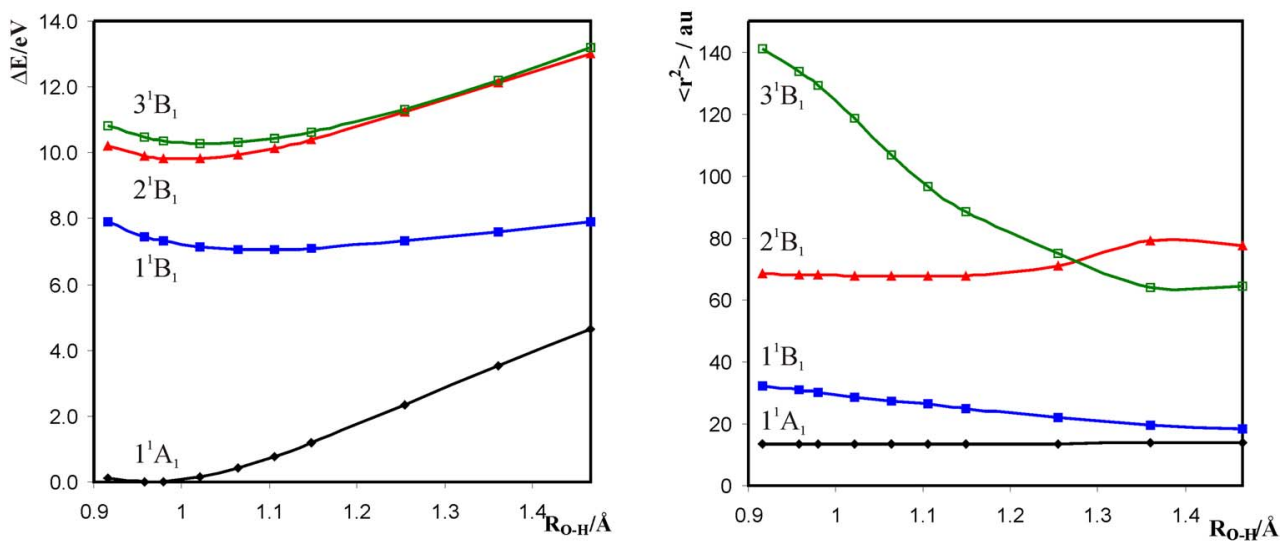

FIG. 1. (Color online) Symmetrical stretching of the $\mathrm{O}-\mathrm{H}$ bonds $(<\mathrm{HOH}$ $\left.104.5^{\circ}\right)$ in the ground and low-lying ${ }^{1} B_{1}$ states of the water molecule. Potential energy curves referred to the corresponding ground-state minimum (left) and computed $\left\langle r^{2}\right\rangle$ values (right). 

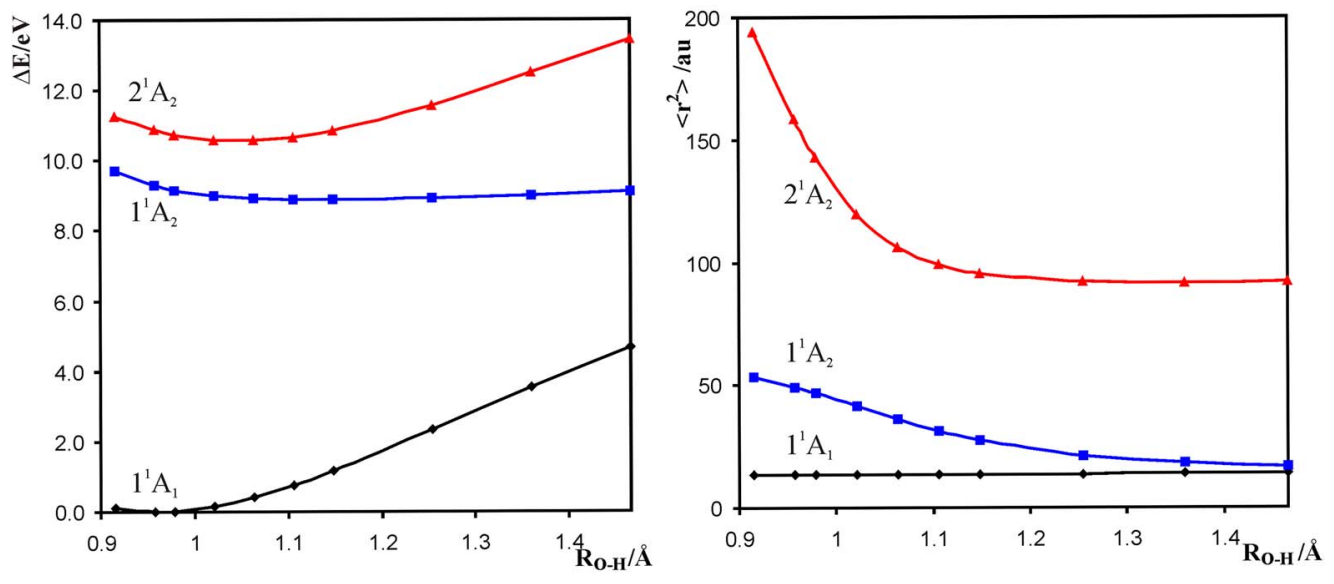

FIG. 2. (Color online) Symmetrical stretching of the $\mathrm{O}-\mathrm{H}$ bonds $(<\mathrm{HOH}$ $\left.104.5^{\circ}\right)$ in the ground and low-lying ${ }^{1} A_{2}$ states of the water molecule. Potential energy curves referred to the corresponding ground-state minimum (left) and computed $\left\langle r^{2}\right\rangle$ values (right). lengthening the $\mathrm{O}-\mathrm{H}$ distance separating this orbital from the related $3 s$-Rydberg orbital. Accordingly, the second ${ }^{1} B_{1}$ root corresponds to the $1 b_{1} \rightarrow 3 s$-Rydberg state at $\mathrm{O}-\mathrm{H}$ distances longer than $1.32 \AA$ (see Table V). The nature of the $1{ }^{1} B_{1}$ state evolves then from a valence-Rydberg mixing type $\left(1 b_{1} \rightarrow 3 s / 4 a_{1}\right)$ at the ground-state equilibrium geometry to a valence character $\left(1 b_{1} \rightarrow 4 a_{1}\right)$ at $\mathrm{O}-\mathrm{H}$ bond lengths around $1.25 \AA$ and longer, which corresponds only to a symmetrical bond stretching of $0.3 \AA$ from the ground-state equilibrium value. The same evolution is expected when stretching only one $\mathrm{O}-\mathrm{H}$ bond length, with the valence character of the state coming from the $\mathrm{O}-\mathrm{H} \sigma^{*}$ orbital, as it has been corroborated by actual calculations. Therefore, the present work confirms definitely that the $1{ }^{1} B_{1}$ state of the water molecule constitutes an example of MO Rydbergization postulated by Mulliken. $^{21,28}$ The reported change in the nature of the state does not arise from a crossing between separated valence and Rydberg states. It exists only one state with changing nature.

The second singlet $1^{1} A_{2}$ excited state is related to a dipole-forbidden transition. Experimental evidence for this state comes from electron-impact spectroscopy and gives an excitation energy of $9.1 \mathrm{eV} .^{16}$ The experimental datum is somewhat lower than the MS-CASPT2 vertical excitation energy, computed to be $9.27 \mathrm{eV}$, and than previous theoretical estimations ${ }^{6,31-33}$ (see Table IV). According to the computed $\left\langle r^{2}\right\rangle$ value, the $1{ }^{1} A_{2}$ state is predicted to be a Rydberg state but with some valence character which occurs at the molecular orbital level, similarly to the $1{ }^{1} B_{1}$ state. In this case, the $3 p_{y}$-Rydberg orbital ( $b_{2}$ symmetry) has certain contribution from the valence antibonding $2 b_{2}$ orbital and the singly excited $1 b_{1} \rightarrow 3 p_{y} / 2 b_{2}$ configuration appears in the computed PMCAS-CI wave function for the $1{ }^{1} A_{2}$ state with a weight of $73 \%$. Two characteristics show the valenceRydberg nature of the $1{ }^{1} A_{2}$ state: first, its computed $\left\langle r^{2}\right\rangle$ has a lower value than the one obtained for the pure $1 b_{1} \rightarrow 3 p_{z}$ Rydberg state (43.2 vs 63.9 a.u.) and second, the $1{ }^{1} A_{2}$ state is separated by $\sim 0.7 \mathrm{eV}$ from the remaining $1 b_{1} \rightarrow 3 p$ Rydberg states, an aspect attributed by Robin ${ }^{14}$ to the stabilization of the $3 p_{y}$ orbital through the mixing with the valence $2 b_{2}$ orbital. Despite both results were also found in the earlier study carried out by Goddard and Hunt, ${ }^{17}$ these authors attributed a Rydberg character to the $1{ }^{1} A_{2}$ state. Further insight has been obtained here by means of the analysis of the effect of the $\mathrm{O}-\mathrm{H}$ stretching on the energy and $\left\langle r^{2}\right\rangle$ of the low-lying ${ }^{1} A_{2}$ states, similarly as it was performed for the ${ }^{1} B_{1}$ state. We have also considered symmetrical $\mathrm{O}-\mathrm{H}$ stretching, keeping fixed the bond angle to the ground-state equilibrium value and preserving thus the $C_{2 v}$ symmetry of the system. Calculations were performed using the basis set $\mathrm{V}$ and the (3141) active space with eight active electrons. The results are shown in Fig. 2 and Table $\mathrm{V}$ for the longest $\mathrm{O}-\mathrm{H}$ distance considered. As can be deduced from the $\left\langle r^{2}\right\rangle$ values included in Fig. 2, the diffuse nature of the $1{ }^{1} A_{2}$ state decreases when increasing the $\mathrm{O}-\mathrm{H}$ distance, while the second computed ${ }^{1} A_{2}$ state is a Rydberg state at all the distances considered but varies the size of the Rydberg orbital. The evolution of the $1{ }^{1} A_{2}$ state with the $\mathrm{O}-\mathrm{H}$ distance, as well as the origin of the transformation found, are the same as those described for the $1{ }^{1} B_{1}$ state, but affecting now the valence antibonding $2 b_{2}$ orbital and the $3 p_{y}$-Rydberg orbital. The nature of the $1^{1} A_{2}$ state evolves then from a valence-Rydberg mixing $\left(1 b_{1} \rightarrow 3 p_{y} / 2 b_{2}\right)$ at geometries close to the groundstate equilibrium geometry to a valence character $\left(1 b_{1}\right.$ $\rightarrow 2 b_{2}$ ) at $\mathrm{O}-\mathrm{H}$ distances longer than $\sim 1.217 \AA$ where the second ${ }^{1} A_{2}$ state becomes the $1 b_{1} \rightarrow 3 p_{y}$ Rydberg state (see Table V). As shown in Fig. 2, the potential energy curve computed for the $1{ }^{1} A_{2}$ state is very flat and shows a minimum at $\mathrm{O}-\mathrm{H}$ distances around $1.11-1.16 \AA$. Symmetrically stretching the $\mathrm{O}-\mathrm{H}$ bond length by $\sim 30 \%$ from its equilibrium value transforms the $1^{1} A_{2}$ state in a pure valence excited state. This state represents therefore another example of Rydbergization at the MO level, ${ }^{21,28}$ and such change in nature does not come from any crossing between the computed ${ }^{1} A_{2}$ potential energy curves, as shown in Fig. 2.

The second band of the electronic spectrum of water in the gas phase appears as a broad and continuous absorption covering the energy range $8.6-10.0 \mathrm{eV}$ and peaking at $9.7 \mathrm{eV} .{ }^{4,11,12}$ The band has superposed a long vibrational progression attributed firstly to the bending mode ${ }^{11,27}$ and more recently to both bending and symmetrical stretching vibrational modes. ${ }^{4}$ On the basis of the vibrational analysis of the band and the earlier $a b$ initio calculations, ${ }^{17,18}$ it was assigned to the so-called $B$ state, corresponding to the $2{ }^{1} A_{1}$ state mainly characterized by the $3 a_{1} \rightarrow 3 s$ excitation. The long vibrational progression on the bending mode indicates an important change in the bond angle upon excitation, an effect which is consistent with electron promotion from the $3 a_{1}$ orbital. ${ }^{11}$ Calculations on the bending potential energy 

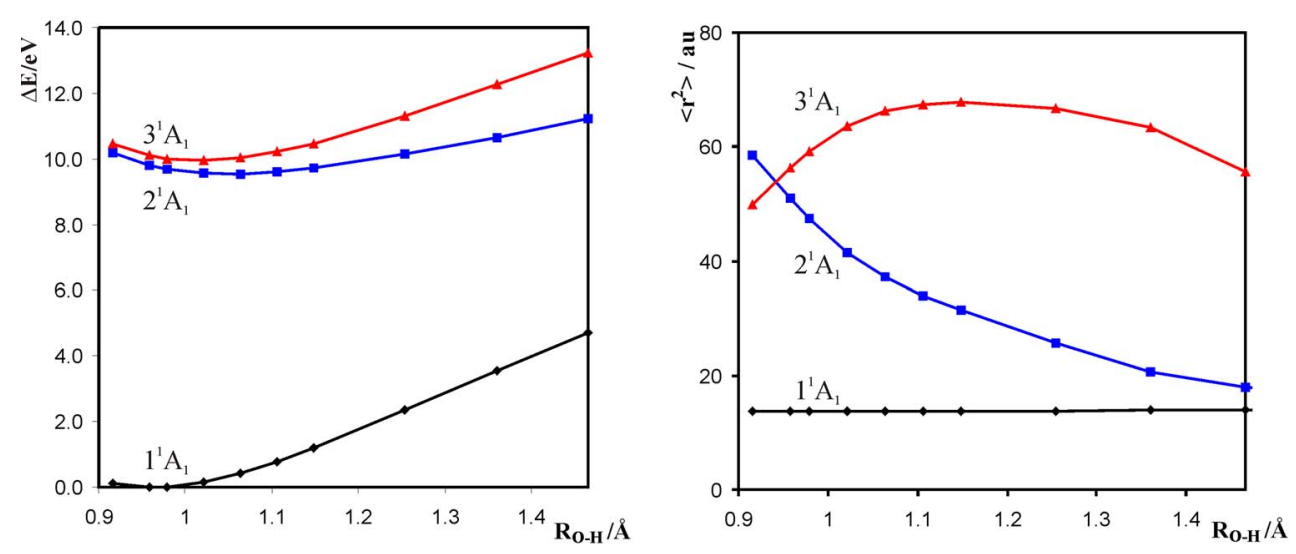

FIG. 3. (Color online) Symmetrical stretching of the $\mathrm{O}-\mathrm{H}$ bonds $(<\mathrm{HOH}$ $\left.104.5^{\circ}\right)$ in the ground and low-lying ${ }^{1} A_{1}$ states of the water molecule. Potential energy curves referred to the corresponding ground-state minimum (left) and computed $\left\langle r^{2}\right\rangle$ values (right). curves predicted a linear geometry for the $2{ }^{1} A_{1}$ state. ${ }^{29,33,52}$ On the other hand, excitation to the second band has been established to lead also to photodissociation through a more complex mechanism ${ }^{33,51,52}$ than in the lowest absorption band. ${ }^{24}$ As shown in Table III, the MS-CASPT2 results predict the $2{ }^{1} A_{1}$ state to be situated vertically at $9.86 \mathrm{eV}$ above the ground state and support its assignment to the second band of the spectrum. Mota et al. ${ }^{4}$ have recently reported an experimental value of $9.991 \mathrm{eV}$ for the origin of the Rydberg series arising from excitation from the $3 a_{1}$ orbital. Although it is unclear for us from their discussion, ${ }^{4}$ it seems that they attribute the second band of the spectrum to a ${ }^{1} A_{1}$ state with valence character and place the ${ }^{1} A_{1}\left(3 a_{1} \rightarrow 3 s\right)$ Rydberg state on the high-energy side of the band. Such interpretation would be wrong according to the present results and previous theoretical studies ${ }^{31-33}$ (see Table IV) since theory predicts only one ${ }^{1} A_{1}$ state in the energy range of the second absorption band. Although we have characterized the $2{ }^{1} A_{1}$ state mainly by means of the $3 a_{1} \rightarrow 3 s / 4 a_{1}$ promotion, this state is described by a mixing of the singly excited $3 a_{1} \rightarrow 3 s / 4 a_{1}$ and $1 b_{1} \rightarrow 3 p_{x}$ configurations, with weights of $70 \%$ and $28 \%$, respectively. The computed $\left\langle x^{2}\right\rangle,\left\langle y^{2}\right\rangle$, and $\left\langle z^{2}\right\rangle$ values for the $2{ }^{1} A_{1}$ state reflect the configurational mixing (see Table III). As discussed above, the $2{ }^{1} A_{1}$ state is then closely related to the $3{ }^{1} A_{1}$ state which is characterized by the same configurations but in a different proportion (weights of $69 \%$ and $28 \%$ for the $1 b_{1} \rightarrow 3 p_{x}$ and $3 a_{1} \rightarrow 3 s / 4 a_{1}$ one-electron promotions, respectively). A similar description for the $2{ }^{1} A_{1}$ and $3{ }^{1} A_{1}$ states was also reported in previous theoretical studies with MRCI methodologies. ${ }^{8,33,55}$ It should be noted here that the one-electron $1 b_{1} \rightarrow 3 p_{x}$ promotion corresponds to a pure Rydberg excitation since the molecule lacks virtual valence orbitals of $b_{1}$ symmetry. In contrast, as indicated by the notation used, the $3 a_{1} \rightarrow 3 s / 4 a_{1}$ excitation has a valenceRydberg mixed character because of the nature of the excited orbital. The configurational mixing increases therefore the Rydberg character of the $2{ }^{1} A_{1}$ state at the ground-state equilibrium geometry, as reflected by the computed $\left\langle r^{2}\right\rangle$. However, some valence contribution in the $B 2{ }^{1} A_{1}$ state, ascribed to the $3 s / 4 a_{1}$ orbital, is still present and it has been suggested from the experimental studies about photodissociation processes of water. ${ }^{23}$ As it was found for the $1{ }^{1} B_{1}$ state (see Fig. 1), the excited orbital $\left(3 s / 4 a_{1}\right)$ is expected to increase its valence character when elongating the $\mathrm{O}-\mathrm{H}$ bond. This evolution of the $3 s / 4 a_{1}$ orbital was obtained in an earlier study of the $2{ }^{1} A_{1}$ state performed at the SCF level by Horsley and Fink. ${ }^{29}$ The authors classified then the state as a semi-Rydberg state following Mulliken's description. ${ }^{21}$ To our knowledge, no further analysis of the nature of the ${ }^{1} A_{1}$ states have been reported, although their potential energy curves have been computed. ${ }^{33,52,56}$ To shed more light into the nature of the low-lying ${ }^{1} A_{1}$ states, we have computed the three lowest ${ }^{1} A_{1}$ states at $C_{2 v}$ geometries with $\mathrm{O}-\mathrm{H}$ distances going from 0.916 to $1.466 \AA$ and focused our attention on the variations of the computed $\left\langle r^{2}\right\rangle$ values with the $\mathrm{O}-\mathrm{H}$ distance. Calculations were carried out using the basis set $\mathrm{V}$ and the active space (4220), namely, the minimal active space required to describe the states of interest, and the bond angle was fixed to the ground-state equilibrium value. The results are collected in Fig. 3 and Table V. The evolution of the computed $\left\langle r^{2}\right\rangle$ for the $2{ }^{1} A_{1}$ state shows that the valence character of the state increases when stretching the $\mathrm{O}-\mathrm{H}$ bonds. It is due to the enlargement of the weight of the singly excited $3 a_{1} \rightarrow 3 s / 4 a_{1}$ configuration in the PMCAS-CI wave function of the $2{ }^{1} A_{1}$ state when elongating the $\mathrm{O}-\mathrm{H}$ bond together with the transformation of the excited orbital toward a valence $4 a_{1}$ orbital. For instance, the $3 a_{1} \rightarrow 3 s / 4 a_{1}$ configuration appears with a weight of $82 \%$ at $r_{\mathrm{O}-\mathrm{H}}=1.064 \AA$ ( $14 \%$ for the singly excited $1 b_{1} \rightarrow 3 p_{x}$ configuration), that is, near to the minimum of the potential energy curve of the $2^{1} A_{1}$ state (see Fig. 3). As expected, the reduction of the configurational mixing as increasing the $\mathrm{O}-\mathrm{H}$ distance also affects the $3{ }^{1} A_{1}$ state and it is reflected in the evolution of the computed $\left\langle r^{2}\right\rangle$ values. Thus, the $3{ }^{1} A_{1}$ state becomes progressively a pure $1 b_{1} \rightarrow 3 p_{x}$-Rydberg state when lengthening the $\mathrm{O}-\mathrm{H}$ bond in the distance range considered. In summary, the present calculations have characterized the $2{ }^{1} A_{1}$ state as a valence-Rydberg state at the ground-state equilibrium geometry and they have shown that the mixing is not only due to the nature of the excited orbital $\left(3 s / 4 a_{1}\right)$ but also to configurational mixing involving the Rydberg $1 b_{1} \rightarrow 3 p_{x}$ excitation. The situation is then different from that found for the two lowest singlet excited states $\left(1{ }^{1} B_{1}\right.$ and $\left.1{ }^{1} A_{2}\right)$ where the valence-Rydberg character arises from MO Rydbergization. On the other hand, the $3{ }^{1} A_{1}$ state can be considered a Rydberg state with a somewhat minor valence character.

Regarding the higher-energy electronic spectrum of gasphase water, it consists of a series of sharp peaks in the $10.0-10.8 \mathrm{eV}$ energy range. ${ }^{4,11,12}$ The $1 b_{1} \rightarrow 3 p_{z}$ and $1 b_{1}$ 
$\rightarrow 3 p_{x}$ transitions have been recorded at 10.01 and $10.16 \mathrm{eV}$ in a recently reported high resolution spectrum, ${ }^{4}$ in agreement with previous experimental determinations. ${ }^{11,12,16}$ These transitions are related to the so-called $\widetilde{C}$ and $\widetilde{D}$ states, ${ }^{27}$ which correspond, respectively, to the $2{ }^{1} B_{1}$ and $3{ }^{1} A_{1}$ states. The $1 b_{1} \rightarrow 4 s$ transition has also been placed in this energy range around $10.6 \mathrm{eV} .{ }^{4,12}$ As shown in Table III, the vertical excitation energies computed for the $2{ }^{1} B_{1}$ and $3{ }^{1} A_{1}$ states are 9.95 and $10.15 \mathrm{eV}$, respectively, confirming the assignment of the observed peaks. ${ }^{4,11,12,16}$ The oscillator strength for the $1{ }^{1} A_{1} \rightarrow 2{ }^{1} B_{1}$ transition is computed to be 0.006 , in agreement with recent high-level theoretical determinations. ${ }^{8,31}$ This low value is consistent with the Rydberg nature of the state and with the selection rule $\Delta 1$ $= \pm 1$ for atomic Rydberg transitions which can be applied here in an approximate way because the $1 b_{1}$ orbital is closely related to an oxygen $2 p$ orbital. The rule explains, for instance, the higher intensity computed for the $1 b_{1} \rightarrow 3 d$ transitions (see Table III). On the other hand, the transition to the $\widetilde{D} 3{ }^{1} A_{1}$ state is predicted here to have the same intensity as the $1{ }^{1} A_{1} \rightarrow 2{ }^{1} A_{1}$ transition, in contrast to the results obtained at the coupled-cluster and CAS/MRCI level which characterize the $1{ }^{1} A_{1} \rightarrow 2{ }^{1} A_{1}$ transition as the most intense one ${ }^{8,31}$ but quite similar to the $1{ }^{1} A_{1} \rightarrow 3{ }^{1} A_{1}$ transition (values of 0.055 and 0.047 at the CAS/MRCI level ${ }^{8}$ ). The computed oscillator strengths are, in general, of the same order of magnitude than the experimental values (see Refs. 7 and 8 for a detailed analysis).

The $1 b_{1} \rightarrow 3 d$ Rydberg transitions have been experimentally located in the $10.8-11.2 \mathrm{eV}$ energy range, ${ }^{11-14,16}$ which is definitely confirmed by the present MS-CASPT2 results (see Table III). Thus, the computed $\left\langle r^{2}\right\rangle$ values as well as the directionality of the electronic charge distribution characterize the singlet excited states calculated between 10.8 and $11.2 \mathrm{eV}$ as the five components of the $1 b_{1} \rightarrow 3 d$ Rydberg promotions. The dipole-forbidden transition to the $2{ }^{1} A_{2}$ state, related to the $1 b_{1} \rightarrow 3 d_{y z}$ one-electron promotion, is computed vertically at $10.84 \mathrm{eV}$. The $2{ }^{1} A_{2}$ state has been detected by electron-impact spectroscopy which has provided an excitation energy of $10.84 \mathrm{eV},{ }^{16}$ matching the present MS-CASPT2 result. Gurtler et al. ${ }^{12}$ assigned the sharp and structured bands at around $11.0 \mathrm{eV}$ to the four optically allowed $1 b_{1} \rightarrow 3 d$ transitions on the basis of their rotational contours. Assignments based on the defect quantum number analysis also situate the $3 d$-members of the Rydberg series converging to the lowest ionization potential at these energies. ${ }^{11,13}$ In particular, Ishiguro et al. ${ }^{13}$ recorded three bands with maximum at $10.99,11.05$, and $11.13 \mathrm{eV}$ which were ascribed to the optically allowed $1 b_{1} \rightarrow 3 d$ Rydberg states. According to the present calculations, these three bands correspond, respectively, to the ${ }^{1} B_{1}\left(1 b_{1} \rightarrow 3 d\right)$, $1{ }^{1} B_{2}\left(1 b_{1} \rightarrow 3 d_{x y}\right)$, and both ${ }^{1} A_{1}\left(1 b_{1} \rightarrow 3 d_{x z}\right)$ and ${ }^{1} B_{1}\left(1 b_{1}\right.$ $\rightarrow 3 d$ ) states.

The $3 a_{1} \rightarrow 3 p$ Rydberg transitions are related to states of ${ }^{1} B_{2}\left(3 a_{1} \rightarrow 3 p_{y}\right),{ }^{1} B_{1}\left(3 a_{1} \rightarrow 3 p_{x}\right)$, and ${ }^{1} A_{1}\left(3 a_{1} \rightarrow 3 p_{z}\right)$ symmetries. As can be seen in Table III, they are computed to be vertically at $11.69,12.13$, and $12.21 \mathrm{eV}$ above the ground state. The $2{ }^{1} B_{2}$ state appears well separated, $0.45 \mathrm{eV}$ lower in energy, from the other two $3 a_{1} \rightarrow 3 p$ states. Moreover, the computed $\left\langle r^{2}\right\rangle$ for the $2{ }^{1} B_{2}$ state is lower than the $\left\langle r^{2}\right\rangle$ values obtained for the ${ }^{1} B_{1}\left(3 a_{1} \rightarrow 3 p_{x}\right)$ and ${ }^{1} A_{1}\left(3 a_{1} \rightarrow 3 p_{z}\right)$ states (54.8 vs 73.4 and 67.5 a.u., respectively). These two features point out to the mixed valence-Rydberg nature of the $2{ }^{1} B_{2}$ state. The mixing of the $3 p_{y}$-Rydberg orbital and the valence antibonding $2 b_{2}$ orbital, allowed by symmetry, is responsible for the valence-Rydberg character, as revealed by the analysis of the PMCAS-CI wave function of the $2{ }^{1} B_{2}$ state. The situation is therefore similar to that found for the $1{ }^{1} A_{2}\left(1 b_{1}\right.$ $\left.\rightarrow 3 p_{y} / 2 b_{2}\right)$ state. The partial valence character can also explain the relative high intensity predicted for the $1^{1} A_{1}$ $\rightarrow 2{ }^{1} B_{2}$ transition. Compared to the oscillator strengths computed for the $3 a_{1} \rightarrow 3 p_{x}$ and $3 a_{1} \rightarrow 3 p_{z}$ Rydberg transitions, that of the $1{ }^{1} A_{1} \rightarrow 2{ }^{1} B_{2}$ transition is more than ten times larger. Experimental evidence for the $2{ }^{1} B_{2}$ state comes from a study performed by Dutuit et al. ${ }^{23}$ These authors proposed the $2{ }^{1} B_{2}$ state as responsible for the continuous absorption recorded in the energy range $10.0-12.6 \mathrm{eV}$, centered at around $11.5 \mathrm{eV}$. Taking into account the great complexity of the electronic spectrum of water at energies above $11.0 \mathrm{eV},{ }^{12,13}$ together with the low intensity values predicted for the $3 a_{1} \rightarrow 3 p_{x}$ and $3 a_{1} \rightarrow 3 p_{z}$ Rydberg transitions, their experimental locations have not been determined, as far as we know. According to the MS-CASPT2 results, both states are located close to the lowest ionization potential of the molecule $(\sim 12.6 \mathrm{eV}),{ }^{50}$ where the absorption spectrum is dominated by the $1 b_{1} \rightarrow n d$ transitions. ${ }^{20}$

We will finally discuss briefly some aspects about previous theoretical studies on the excited states of water. The most recent and representative results are included in Table IV. When comparing these previous results to the present MS-CASPT2 data, it should be kept in mind that not all of them have used the experimental ground-state equilibrium geometry for the calculations as in our study. An exhaustive recompilation of theoretical data for the excited states of water can be found in a recent study reported by $\mathrm{Li}$ and Paldus. ${ }^{6}$ These authors chose the water molecule to analyze the performance of their multireference coupled-cluster method (MRCC results in Table IV). Similarly, Cai et al. ${ }^{32}$ have studied the excited states of water using time-dependent density functional theory (TD-DFT) in order to determine what characteristics of the potential are required to get results of quality comparable to those obtained with the equation of motion coupled cluster (EOM-CC) and MRCI techniques. The TDDFT values included in Table IV correspond to the best TDDFT results reported by Cai et $a l .{ }^{32}$ and they have been obtained using an asymptotic correction in the density functional, which has been shown to be necessary in order to get reliable results for states with a significant Rydberg character. ${ }^{32}$ For the five lowest singlet excited states, the CCSDR(3) vertical excitation energies (noniterative triples correction to CCSD excitation energies) reported by Christiansen et al. ${ }^{31}$ which probably represent the highest level of theory used, are $0.07-0.16 \mathrm{eV}$ larger than the present MSCASPT2 results. In contrast, the CCSDR(3) vertical excitation energies for the $1 b_{1} \rightarrow 3 d$ Rydberg states are $0.2-0.3 \mathrm{eV}$ overestimated with respect to our MS-CASPT2 results and the experimental data. ${ }^{13} \mathrm{~A}$ better agreement is, however, 
TABLE VI. Computed MS-CASPT2 (MS-PT2) vertical excitation energies (eV) and other properties for the triplet excited states of the water molecule compared to experimental data and previous theoretical results.

\begin{tabular}{lrrrrrrl}
\hline \hline State & MS-PT2 & $\left\langle x^{2}\right\rangle$ & $\left\langle y^{2}\right\rangle$ & $\left\langle z^{2}\right\rangle$ & $\left\langle r^{2}\right\rangle$ & Expt. $(\mathrm{eV})^{\mathrm{a}}$ & Theor. \\
\hline $1{ }^{3} B_{1}\left(1 b_{1} \rightarrow 3 s / 4 a_{1}\right)$ & 7.13 & 8.2 & 9.0 & 10.3 & 27.5 & 7.0 & $7.23,{ }^{\mathrm{b}} 7.18,{ }^{\mathrm{c}} 7.14^{\mathrm{d}}$ \\
$1{ }^{3} A_{2}\left(1 b_{1} \rightarrow 3 p_{y} / 2 b_{2}\right)$ & 9.12 & 9.1 & 20.1 & 10.5 & 39.7 & 8.9 & $9.15,{ }^{\mathrm{b}} 9.17,,^{\mathrm{c}} 9.42^{\mathrm{d}}$ \\
$1{ }^{3} A_{1}\left(3 a_{1} \rightarrow 3 s / 4 a_{1}\right)$ & 9.47 & 9.0 & 9.0 & 9.9 & 27.9 & 9.3 & $9.30,{ }^{\mathrm{b}} 9.47,{ }^{\mathrm{c}} 9.78^{\mathrm{d}}$ \\
$2{ }^{3} A_{1}\left(1 b_{1} \rightarrow 3 p_{x}\right)$ & 9.87 & 35.5 & 12.4 & 14.3 & 62.2 & 9.81 & $9.76,{ }^{\mathrm{b}} 9.76,{ }^{\mathrm{c}} 10.52^{\mathrm{d}}$ \\
$2{ }^{3} B_{1}\left(1 b_{1} \rightarrow 3 p_{z}\right)$ & 9.90 & 15.9 & 15.4 & 30.3 & 61.6 & 9.98 & $9.89,,^{\mathrm{b}} 9.93,{ }^{\mathrm{c}} 10.61^{\mathrm{d}}$ \\
$2{ }^{3} A_{2}\left(1 b_{1} \rightarrow 3 d_{y z}\right)$ & 10.80 & 21.4 & 55.1 & 27.1 & 103.6 & 10.68 & \\
${ }^{3} B_{1}\left(1 b_{1} \rightarrow 3 d\right)$ & 11.01 & 22.8 & 48.6 & 24.2 & 95.6 & & \\
$1^{3} B_{2}\left(1 b_{t} \rightarrow 3 d_{x y}\right)$ & 11.04 & 37.2 & 35.3 & 14.3 & 86.8 & & \\
${ }^{3} A_{1}\left(1 b_{1} \rightarrow 3 d_{x z}\right)$ & 11.10 & 68.1 & 23.2 & 41.8 & 133.1 & & \\
${ }^{3} B_{1}\left(1 b_{1} \rightarrow 3 d\right)$ & 11.16 & 32.0 & 21.3 & 67.1 & 120.4 & & \\
$2{ }^{3} B_{2}\left(3 a_{1} \rightarrow 3 p_{y} / 2 b_{2}\right)$ & 11.41 & 11.2 & 24.0 & 11.0 & 46.2 & & $11.29,{ }^{\mathrm{b}} 11.35,{ }^{\mathrm{c}} 11.41^{\mathrm{d}}$ \\
${ }^{3} A_{1}\left(3 a_{1} \rightarrow 3 p_{z}\right)$ & 11.96 & 15.8 & 14.4 & 27.6 & 57.8 & & \\
${ }^{3} B_{1}\left(3 a_{1} \rightarrow 3 p_{x}\right)$ & 12.08 & 41.6 & 14.1 & 15.1 & 70.8 & & \\
\hline \hline
\end{tabular}

${ }^{\mathrm{a} E l e c t r o n-i m p a c t ~ d a t a ~ f r o m ~ C h u t j i a n ~ e t ~ a l ., ~ R e f . ~} 16$.

${ }^{\mathrm{b}}$ TD-DFT/d-aug-cc-pVTZ results from Cai et al., Ref. 32.

${ }^{c}$ EOM-CCSD/d-aug-cc-pVTZ results from Cai et al., Ref. 32.

${ }^{\mathrm{d}} \mathrm{MRCI}+\mathrm{Q} /$ aug-cc-pVTZ results from Cai et al., Ref. 32.

found between the EOM-CCSD and MS-CASPT2 results (see Table IV). Regarding the MRCI results, apart from those included in Table IV, it should be mentioned here the studies on the oscillator strengths of the low-lying electronic transitions of water performed by Borges ${ }^{7,8}$ using the CAS/MRCI approach. At the highest level of theory used by Borges, ${ }^{7,8}$ the computed vertical excitation energies for the $\widetilde{A}, \widetilde{B}$, and $\widetilde{D}$ states $(7.69,9.92$, and $10.28 \mathrm{eV})$ differ by less than $0.2 \mathrm{eV}$ from the MS-CASPT2 values (see data for the $1{ }^{1} B_{1}, 2{ }^{1} A_{1}$, and $3{ }^{1} A_{1}$ states in Table IV). His results for the remaining states examined show, however, a large difference $(0.3-0.7 \mathrm{eV})$. As a whole, the theoretical values included in Table IV for the five lowest excited states are at most in a $0.3 \mathrm{eV}$ energy interval, except for the MRCI+Q results ${ }^{32}$ which are clearly overestimated for the highest states.

Triplet excited states. The results obtained for the triplet excited states of water at the FC geometry are shown in Table VI. As can be deduced from the results collected, some of the features found for the singlet excited states are also present in the corresponding triplet excited states. In particular, the computed $\left\langle r^{2}\right\rangle$ values for the three lowest triplet states, $1{ }^{3} B_{1}, 1{ }^{3} A_{2}$, and $1{ }^{3} A_{1}$, indicate that these states undergo also MO Rydbergization, as it was determined for the corresponding singlet states (see Table III). It is also noted that the $1{ }^{3} A_{2}\left(1 b_{1} \rightarrow 3 p_{y} / 2 b_{2}\right)$ state appears at $0.7 \mathrm{eV}$ lower in energy than the other two triplet $1 b_{1} \rightarrow 3 p$ Rydberg states, which can be considered as indicative of its valence contribution, as it was discussed for the $1{ }^{1} A_{2}$ state. ${ }^{14}$ As shown in Table VI, such energy difference also occurs for the triplet $3 a_{1} \rightarrow 3 p$ states. The $2{ }^{3} B_{2}$ state, described by the $3 a_{1}$ $\rightarrow 3 p_{y} / 2 b_{2}$ one-electron promotion, is computed to be situated $\sim 0.6 \mathrm{eV}$ below the next ${ }^{3} A_{1}\left(3 a_{1} \rightarrow 3 p_{z}\right)$ state. The computed $\left\langle r^{2}\right\rangle$ for the $2{ }^{3} B_{2}$ state is appreciably lower than those calculated for the ${ }^{3} B_{1}\left(3 a_{1} \rightarrow 3 p_{x}\right)$ and ${ }^{3} A_{1}\left(3 a_{1} \rightarrow 3 p_{z}\right)$ states (46.2 vs 70.8 and 57.8 a.u., respectively). This result suggests a mixed valence-Rydberg nature of the $2{ }^{3} B_{2}$ state, which also arises from MO Rydbergization according to the computed PMCAS-CI wave function.
As could be expected, the computed singlet-triplet energy splittings are, in general, found to be larger $(0.3-0.4 \mathrm{eV})$ for those states having a mixed valenceRydberg nature, except for the $1{ }^{3} A_{2}$ state calculated at $9.12 \mathrm{eV}$, which is $0.15 \mathrm{eV}$ below the corresponding $1{ }^{1} A_{2}$ state (see Table III). The $2{ }^{3} A_{1}\left(1 b_{1} \rightarrow 3 p_{x}\right)$ Rydberg state also has a significant singlet-triplet energy splitting $(0.28 \mathrm{eV})$. The $1 b_{1} \rightarrow 3 d$ Rydberg states show negligible $(\sim 0.01-0.04 \mathrm{eV})$ singlet-triplet energy splittings. On the other hand, unlike the singlet $2{ }^{1} A_{1}$ and $3{ }^{1} A_{1}$ states, no configurational mixing has been found for the related $1^{3} A_{1}$ and $2^{3} A_{1}$ states, as can be inferred from the computed $\left\langle x^{2}\right\rangle,\left\langle y^{2}\right\rangle$, and $\left\langle z^{2}\right\rangle$ values for the $1{ }^{3} A_{1}$ state (see Table VI).

As regards the comparison with the experimental data, it is noted that the MS-CASPT2 vertical excitation energies computed for the three lowest triplet excited states are approximately $0.2 \mathrm{eV}$ overestimated with respect to the electron-impact data reported by Chutjian et al. ${ }^{16}$ The situation is then similar to that found for the singlet excited states with valence-Rydberg character. As can be seen in Table VI, the difference between the MS-CASPT2 results and the experimental data is, however, minor for the three next triplet excited states $\left(2{ }^{3} B_{1}, 2{ }^{3} A_{1}\right.$, and $\left.2{ }^{3} A_{2}\right)$ having all them Rydberg character. Table VI also includes the theoretical values obtained by Cai et al. ${ }^{32}$ for the triplet states using different methods. As a whole, they differ at most $0.3 \mathrm{eV}$ from the MS-CASPT2 vertical excitation energies, except for the MRCI+Q results for the $2{ }^{3} B_{1}$ and $2{ }^{3} A_{1}$ states which are clearly overestimated, as it was found for the corresponding singlet states (see Table IV).

\section{SUMMARY AND CONCLUSIONS}

We have presented here a high-level ab initio study on the excited states of the water molecule using the extended multistate CASPT2 method ${ }^{35,36,45}$ and highly accurate ANOtype basis sets. The study includes the singlet and triplet excited states arising from excitations from the two highest 
occupied molecular orbitals $\left(3 a_{1}\right.$ and $\left.1 b_{1}\right)$ to the valence and $3 s-, 3 p-$, and $3 d$-Rydberg orbitals. The states have been characterized by means of the computed PMCAS-CI wave functions and the second moments of the electronic charge distribution. The computed vertical excitation energies have been discussed in relation to experimental data and previous theoretical results. As a whole, the largest deviation $(\leqslant 0.2 \mathrm{eV})$ from the experimental data has been obtained for the three low-lying singlet and triplet excited states, which can be related to the shape of the recorded bands as well as to the nature of the states. It is concluded that the electronic spectrum of gas-phase water has a predominant Rydberg character at the Franck-Condon geometry, in line with the general view, although a certain degree of valence character has been shown to occur in the three lowest singlet states. Moreover, analysis of the nature of the low-lying excited states through the calculation of a portion of the symmetrical stretching $\mathrm{O}-\mathrm{H}$ potential energy curves has clearly confirmed that the valence-Rydberg mixing of the states results from MO Rydbergization as described by Mulliken. ${ }^{21,28}$ Accordingly, there is only one state whose character changes with the molecular geometry as reflected indeed by all our attempts to find pure valence states at the FC geometry. In particular, for the lowest singlet $\widetilde{A} 1{ }^{1} B_{1}$ excited state, related to the one-electron $1 b_{1} \rightarrow 3 s / 4 a_{1}$ promotion, it has been established that the excited orbital has a substantial $3 s$ Rydberg character at the $\mathrm{FC}$ region but evolves to become a valence antibonding $4 a_{1}$ orbital at elongated $\mathrm{O}-\mathrm{H}$ bond lengths. This behavior is consistent with the photodissociation process into ground-state $\mathrm{H} \cdot$ and $\mathrm{OH} \cdot$ radicals following excitation of water in the energy range of the lowest absorption band. ${ }^{24} \mathrm{~A}$ valence-Rydberg mixing is therefore also expected for those states ascribed to promotions from deeper orbitals to the $3 s / 4 a_{1}$ orbital, as pointed out by Dutuit et al. ${ }^{23}$ and it occurs for the $2{ }^{1} A_{1}\left(3 a_{1} \rightarrow 3 s / 4 a_{1}\right)$ state considered here. Excitation to the $3 p_{y} / 2 b_{2}$ orbital also leads to excited states of valenceRydberg nature, as shown for the $1{ }^{1} A_{2}\left(1 b_{1} \rightarrow 3 p_{y} / 2 b_{2}\right)$ state. Finally, it is also worth mentioning that the electronic spectra of $\mathrm{CH}_{4}$ and $\mathrm{NH}_{3}$ molecules have been also interpreted as arising from Rydberg states at the FC region, having the lowest singlet excited state a valence-Rydberg nature. ${ }^{14,20}$ No valence excited states have been characterized in these molecules at the ground-state equilibrium geometry. As for the water molecule, an increase of the valence character of the lowest singlet excited state occurs in these hydrides upon stretching of one of the bonds. Interestingly, Mulliken ${ }^{21}$ considered the lowest singlet excited state of the three molecules as an example of MO Rydbergization, although his prediction failed for other systems such as ethene. ${ }^{14,45,57}$ The present study has confirmed the MO Rydbergization in the lowest singlet $1{ }^{1} B_{1}$ state of the water molecule. In addition, a similar situation has been found for the $1{ }^{1} A_{2}$ singlet excited state. A more complex behavior has been determined for the $2{ }^{1} A_{1}$ state, where both MO Rydbergization and configurational mixing take place. Therefore, whereas at the ground-state equilibrium distance the three excited states have a predominant diffuse character, they have a clear valence nature upon symmetric stretching of the $\mathrm{O}-\mathrm{H}$ bond, and it is only then when the typical view of states and orbit- als displayed in general chemistry textbooks is applicable. From the overall study, the essentials of the lowest excited states of the water molecule are therefore better understood.

\section{ACKNOWLEDGMENTS}

Financial support is acknowledged from projects CTQ2004-01739 and CTQ2007-61260 of the Spanish MEC/ FEDER and GV06-192 of the Generalitat Valenciana.

${ }^{1}$ R. S. Mulliken, J. Chem. Phys. 3, 506 (1935).

${ }^{2}$ W. C. Price, J. Chem. Phys. 4, 147 (1936).

${ }^{3}$ P. Maksyutenko, J. S. Muenter, N. F. Zobov, S. V. Shirin, O. L. Polyansky, T. R. Rizzo, and O. V. Boyarkin, J. Chem. Phys. 126, 241101 (2007).

${ }^{4}$ R. Mota, R. Parafita, A. Giuliani, M.-J. Hubin-Franskin, J. M. C. Lourenço, G. Garcia, S. V. Hoffmann, N. J. Mason, P. A. Ribeiro, M. Raposo, and P. Limão-Vieira, Chem. Phys. Lett. 416, 152 (2005).

${ }^{5}$ M. López del Puerto, M. L. Tiago, I. Vasiliev, and J. R. Chelikowsky, Phys. Rev. A 72, 052504 (2005).

${ }^{6}$ X. Li and J. Paldus, Mol. Phys. 104, 661 (2006).

${ }^{7}$ I. Borges, Jr., J. Phys. B 39, 641 (2006).

${ }^{8}$ I. Borges, Jr., Chem. Phys. 328, 284 (2006).

${ }^{9}$ K. Watanabe and A. S. Jursa, J. Chem. Phys. 41, 1650 (1964).

${ }^{10}$ D. H. Katayama, R. E. Huffman, and C. L. O'Bryan, J. Chem. Phys. 59, 4309 (1973).

${ }^{11}$ H. T. Wang, W. S. Felps, and S. P. McGlynn, J. Chem. Phys. 67, 2614 (1977).

${ }^{12}$ P. Gürtler, V. Saile, and E. E. Koch, Chem. Phys. Lett. 51, 386 (1977).

${ }^{13}$ E. Ishiguro, M. Sasanuma, H. Masuko, Y. Morioka, and M. Nakamura, J. Phys. B 11, 993 (1978).

${ }^{14}$ M. B. Robin, Higher Excited States of Polyatomic Molecules (Academic, New York, 1985), Vol. 3.

${ }^{15}$ S. Trajmar and R. I. Hall, J. Phys. B 7, L458 (1974).

${ }^{16}$ A. Chutjian, R. I. Hall, and S. Trajmar, J. Chem. Phys. 63, 892 (1975).

${ }^{17}$ W. A. GoddardIII and W. J. Hunt, Chem. Phys. Lett. 24, 464 (1974).

${ }^{18}$ N. W. Winter, W. A. Goddard III, and F. W. Bobrowicz, J. Chem. Phys. 62, 4325 (1975).

${ }^{19}$ R. J. Buenker and S. D. Peyerimhoff, Chem. Phys. Lett. 29, 253 (1974).

${ }^{20}$ M. N. R. Ashfold and S. R. Langford, in The Role of Rydberg States in Spectroscopy and Photochemistry, edited by C. Sándorfy (Kluwer, Dordrecht, 1999), Chap. 2, p. 23.

${ }^{21}$ R. S. Mulliken, Acc. Chem. Res. 9, 7 (1976).

${ }^{22}$ G. H. F. Diercksen, W. P. Kraemer, T. N. Rescigno, C. F. Bender, B. V. Mckoy, S. R. Langhoff, and P. W. Langhoff, J. Chem. Phys. 76, 1043 (1982).

${ }^{23}$ O. Dutuit, A. Tabche-Fouhaile, I. Nenner, H. Frohlich, and P. M. Guyon, J. Chem. Phys. 83, 584 (1985).

${ }^{24}$ V. Engel, V. Staemmler, R. L. Vander Wal, F. F. Crim, R. J. Sension, B. Hudson, P. Andresen, S. Hennig, K. Weide, and R. Schinke, J. Phys. Chem. 96, 3201 (1992).

${ }^{25}$ X. F. Yang, D. W. Hwang, J. J. Lin, and X. Ying, J. Chem. Phys. 113, 10597 (2000).

${ }^{26}$ R. van Harrevelt and M. C. van Hemert, J. Chem. Phys. 114, 9453 (2001).

${ }^{27}$ G. Herzberg, Molecular Spectra and Molecular Structure III (van Nostrand, New York, 1966).

${ }^{28}$ R. S. Mulliken, Chem. Phys. Lett. 46, 197 (1977).

${ }^{29}$ J. A. Horsley and W. H. Fink, J. Chem. Phys. 50, 750 (1969).

${ }^{30}$ M. Chergui and N. Schwentner, Chem. Phys. Lett. 219, 237 (1994).

${ }^{31}$ O. Christiansen, T. M. Nymand, and K. V. Mikkelsen, J. Chem. Phys. 113, 8101 (2000).

${ }^{32}$ Z. Cai, D. J. Tozer, and J. R. Reimers, J. Chem. Phys. 113, 7084 (2000).

${ }^{33}$ R. van Harrevelt and M. C. van Hemert, J. Chem. Phys. 112, 5777 (2000).

${ }^{34}$ D. M. Chipman, J. Chem. Phys. 122, 044111 (2005).

${ }^{35}$ K. Andersson, P.-Å. Malmqvist, B. O. Roos, A. J. Sadlej, and K. Wolinski, J. Phys. Chem. 94, 5483 (1990).

${ }^{36}$ K. Andersson, P.-A. Malmqvist, and B. O. Roos, J. Chem. Phys. 96, 1218 (1992).

${ }^{37}$ B. O. Roos, K. Andersson, M. P. Fülscher, P.-A. Malmqvist, L. SerranoAndrés, K. Pierloot, and M. Merchán, Adv. Chem. Phys. 93, 219 (1996).

${ }^{38}$ M. Merchán, L. Serrano-Andrés, M. P. Fülscher, and B. O. Roos, in 
Recent Advances in Multireference Methods, edited by K. Hirao (World Scientific, Singapore, 1999).

${ }^{39}$ L. Serrano-Andrés and M. Merchán, in Encyclopedia of Computational Chemistry, edited by P. v. R. Schleyer, P. R. Schreiner, H. F. Schaefer III, W. L. Jorgensen, W. Thiel, and R. C. Glen (Wiley, Chichester, 2004).

${ }^{40}$ M. Merchán and L. Serrano-Andrés, in Computational Photochemistry, edited by M. Olivucci (Elsevier, Amsterdam, 2005), pp. 35-91.

${ }^{41}$ B. O. Roos, in Advances in Chemical Physics: Ab Initio Methods in Quantum Chemistry II, edited by K. P. Lawley (Wiley, Chinchester, 1987), Chap. 69, p. 399.

${ }^{42}$ S. Klein, E. Kochanski, A. Strich, and A. J. Sadlej, Theor. Chim. Acta 94, 75 (1996).

${ }^{43}$ P.-O. Widmark, P.-Å. Malmqvist, and B. O. Roos, Theor. Chim. Acta 77, 291 (1990).

${ }^{44}$ N. Forsberg and P.-Å. Malmqvist, Chem. Phys. Lett. 274, 196 (1997).

${ }^{45}$ J. Finley, P.-A. Malmqvist, B. O. Roos, and L. Serrano-Andrés, Chem. Phys. Lett. 288, 299 (1998).

${ }^{46}$ R. Crespo, M. Merchán, and J. Michl, J. Phys. Chem. A 104, 8593 (2000).

${ }^{47}$ P.- $\AA$. Malmqvist, Int. J. Quantum Chem. 30, 479 (1986).

${ }^{48}$ P.-Å. Malmqvist and B. O. Roos, Chem. Phys. Lett. 155, 189 (1989).

${ }^{49}$ K. Andersson, M. Barysz, A. Bernhardsson, M. R. A. Blomberg, Y. Carissan, D. L. Cooper, M. Cossi, M. P. Fülscher, L. Gagliardi, C. de
Graaf, B. Hess, G. Hagberg, G. Karlström, R. Lindh, P.-A. Malmqvist, T. Nakajima, P. Neogrády, J. Olsen, J. Raab, B. O. Roos, U. Ryde, B. Schimmelpfennig, M. Schütz, L. Seijo, L. Serrano-Andrés, P. E. M. Siegbahn, J. Stålring, T. Thorsteinsson, V. Veryazov, and P.-O. Widmark, MOLCAS, version 6.0, Department of Theoretical Chemistry, Chemical Centre, University of Lund, Sweden, 2004.

${ }^{50}$ L. Karlsson, L. Mattson, R. Jadrny, R. G. Albridge, S. Pinchas, T. Bergmark, and K. Siegbahn, J. Chem. Phys. 62, 4745 (1975).

${ }^{51}$ J. H. Fillion, R. van Harrevelt, J. Ruiz, M. Castillejo, A. H. Zanganeh, J. L. Lemaire, M. C. van Hemert, and F. Rostas, J. Phys. Chem. A 105, 11414 (2001)

${ }^{52}$ G. Theodorakopoulos, I. D. Petsalakis, R. J. Buenker, and S. D. Peyerimhoff, Chem. Phys. Lett. 105, 253 (1984).

${ }^{53}$ W. F. Chan, G. Cooper, and C. E. Brion, Chem. Phys. 178, 387 (1993).

${ }^{54}$ C. Makochekanwa, R. Kajita, H. Kato, M. Kitajima, H. Cho, M. Kimura, and H. Tanaka, J. Chem. Phys. 122, 014314 (2005).

${ }^{55}$ G. Theodorakopoulos, C. A. Nicolaides, R. J. Buenker, and S. D. Peyerimhoff, Chem. Phys. Lett. 89, 164 (1982).

${ }^{56}$ F. Schneider, F. Di Giacomo, and F. A. Gianturco, J. Chem. Phys. 104, 5153 (1996)

${ }^{57}$ L. Serrano-Andrés, M. Merchán, I. Nebot-Gil, R. Lindh, and B. O. Roos, J. Chem. Phys. 98, 3151 (1993). 